\title{
Research of Energy Consumption Prediction of Paper Enterprises Based on GA-BP Neural Network Algorithm
}

\author{
Yanan $\mathrm{Hu}^{1, \mathrm{a} *}$, Jiaofei Huo ${ }^{2, \mathrm{~b}}$ and Pengwen Wang ${ }^{3, \mathrm{c}}$ \\ Mechanical and electrical technology department \\ Xijing University, Shaanxi Xi'an, China \\ a2664961273@qq.com
}

*The Corresponding author

Keywords: Energy management system; B. Data; BP neural network; Genetic algorithm; Weights and Threshold.

\begin{abstract}
Aiming at the problem of high energy consumption and low utilization rate and low energy consumption management in paper making industry, The Energy management system (EMS)of based on SIEMENS B. Data software is proposed. Because BP neural network has its own defects, which leads to the problem of inaccurate prediction model. In order to obtain the optimum network model, using genetic algorithm to optimize BP neural network weights and threshold. Simulation results show that: GA-BP neural network model has the advantages of high speed and high precision. The energy management system has been successfully applied to a paper making industry in Shandong, and the system has the functions of monitoring and management, energy consumption data analysis and prediction, energy network optimization. The system can meet the requirements of energy management and energy saving in papermaking enterprises.
\end{abstract}

\section{Introduction}

Due to the weak foundation and backward management, China's pulp and paper industry is still an extensive production mode with high energy consumption and high pollution. Energy consumption and environmental problems have become an urgent problem for enterprises and government. EMS is an effective way to solve the problem of energy consumption and environmental pollution reduction [1].

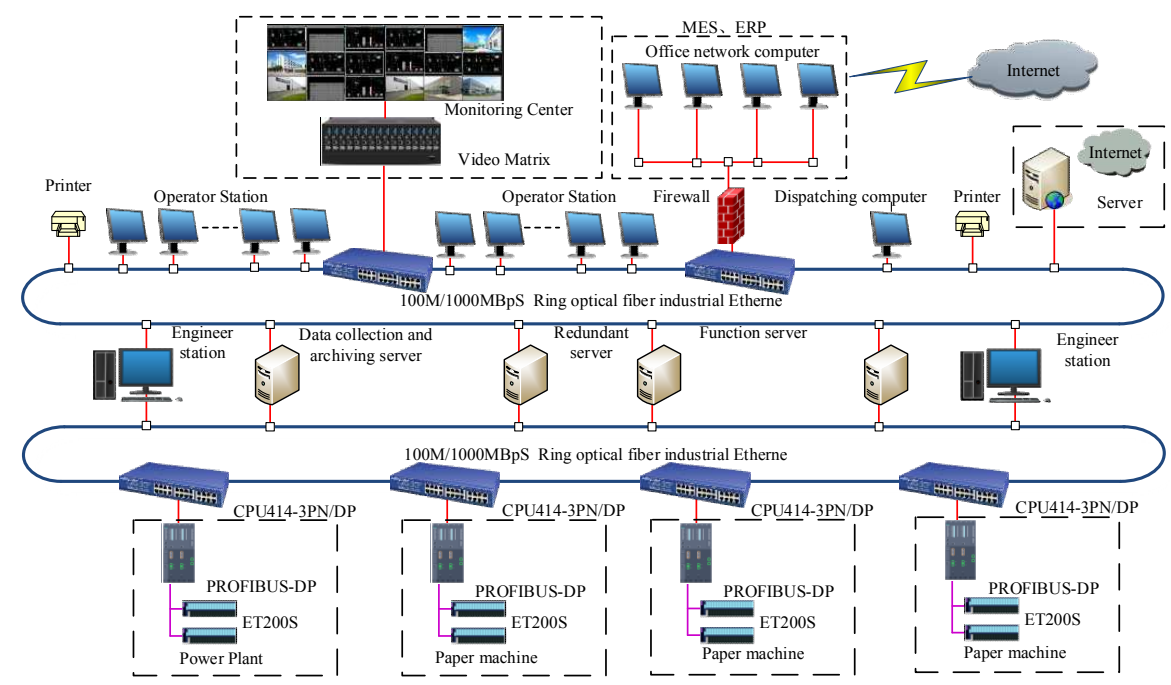

Figure 1. Finite Structure of communication network

\section{EMS System Architecture}

System Architecture. According to the energy consumption characteristics and the needs of enterprises in Shandong of China, the system framework is designed as three layers: data collection layer, data processing layer and application management layer. The data acquisition layer transfers 
the energy consumption data from the production process to the energy management system. The data processing layer mainly uses the OPC technology to process the real-time energy consumption data in PLC, WinCC and B.Data software database[2]. Application management mainly realizes the function of data analysis, prediction, optimization and management.

System Network Architecture. As shown in Fig. 1, the architecture of the network management system is based on the redundant network server, which greatly improves the communication ability of the system and the reliability of the system. Based on the Oracle database management platform with SIEMENS B.Data energy data preprocessing, data analysis and management of energy consumption, energy balance, energy accounting, energy planning, energy control, energy consumption report production, project management and other functions[3].

\section{GA -BP Neural Network Prediction Model}

GA-BP Neural Network Model. BP neural network has the advantages of self-learning, but also has the disadvantage of easily falling into local minima. GA is a large area search from the string set to avoid the risk of falling into the local optimal solution, which is beneficial to the global optimization. This paper combines the advantages of BP neural network and GA algorithm, uses GA algorithm to optimize the weights and thresholds of BP neural network model[4]. As shown in Fig. 2 , the GA-BP optimization network model is established in this paper.

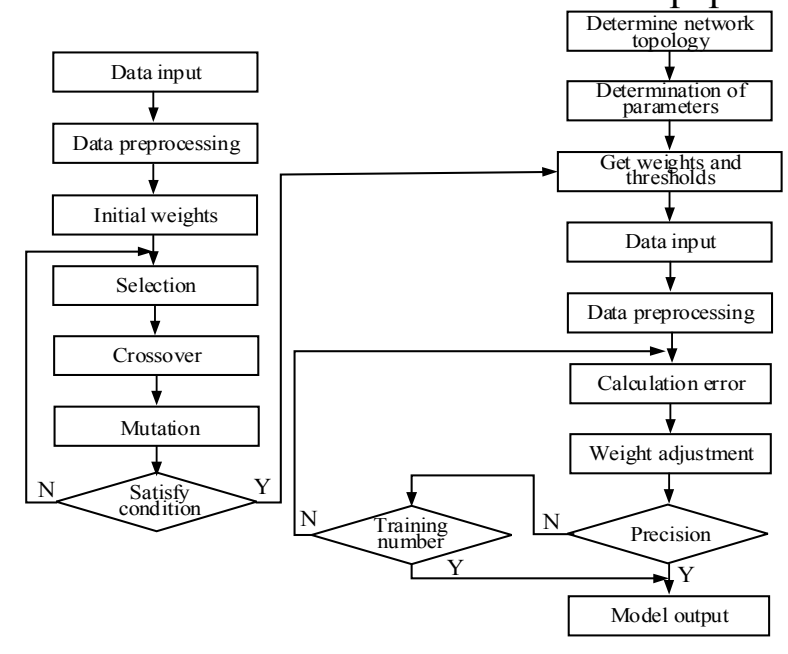

Figure 2. Finite GA-BP neural network model

GA-BP Algorithm Design. 1) Coding mode. In this paper, the weights and thresholds of BP neural network are coded into a long string of real numbers. The string is $\zeta=\left[\mathrm{V}_{1}, \mathrm{~W}_{1}, \mathrm{~B}_{1}, \mathrm{~B}_{2}\right], \mathrm{V}_{1}$ is the weights between the hidden layer and the input layer, $W_{1}$ is the weight between the output layer and the hidden layer, $\mathrm{B}_{1}$ is the threshold value of the hidden layer neuron, $\mathrm{B}_{2}$ is the threshold value of the output layer neuron. 2) Selection, crossover and mutation. In this paper, the selection operator uses roulette selection method. The reason for the inverse of fitness value is that the greater the fitness, the smaller the probability of being selected. Crossover is the key to ensure good traits can be inherited[5]. It is randomly exchanges two genes in the population according to the crossover rate, which can generate new gene combinations[6]. The mutation is the mutation of the gene values on some loci of the individual strings in the population.

\section{Establishment and Simulation of Prediction Model}

System Prediction Model. The first 22 days of production energy consumption data are used to train the GA-BP model. Then, the remaining six days of this month's energy consumption forecast data obtained based on GA-BP model. In the MATLAB software, the data used to train the neural network is assigned to the matrix variable input_train. The actual production of the 88 sets of data assigned to the matrix variable output_train. The 24 sets of input and output data for testing are 
assigned to matrix input test and output_test. Finally, we use mapminmax () function to normalize the data. The normalization procedure is as follows[7]:

[inputn,inputps] $=$ mapminmax(input_train);

[outputn,outputps]= mapminmax(output_train);

The weights and thresholds are optimized by genetic algorithm to BP network. To train the network in terms of actual power consumption and actual production. The activation function of the hidden layer and output layer selects tan.sigrnoid type function. The termination condition of network learning is the error precision $\mathrm{E}<0.00001$ or the maximum training number is 100 times. The initial learning rate is 0.1 . The training program code is as follows[8]:

net.trainParam.epochs $=100$; net.trainParam. $1 \mathrm{r}=0.1$;

net.trainParam.goal $=0.00001 ;$ an $=\operatorname{sim}($ net,inputn_test);

Bpoutput=mapminmax ('reverse', an,outputps);

Comparative Analysis of Simulation Results. The optimal individual fitness value is shown in Fig.3. With the development of evolutionary algebra, the optimal individual fitness value of each generation is gradually reduced until the fiftieth generation.

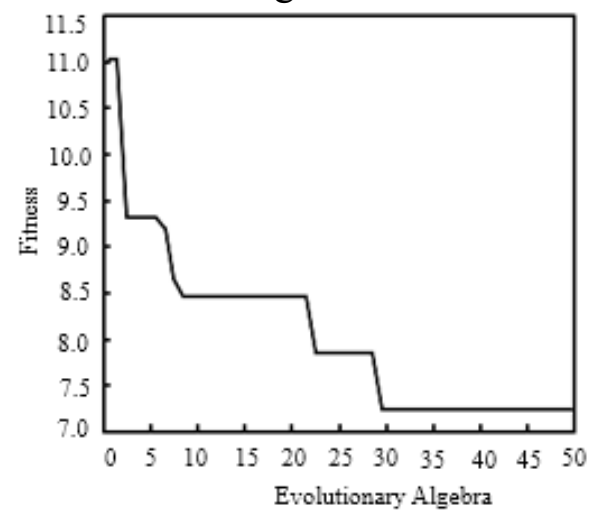

Figure 3. Finite Optimal individual fitness value trend chart

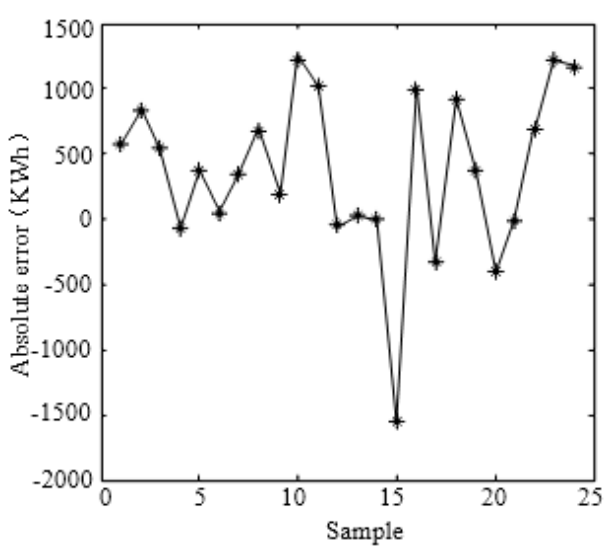

Figure 4. Finite Standard BP error diagram

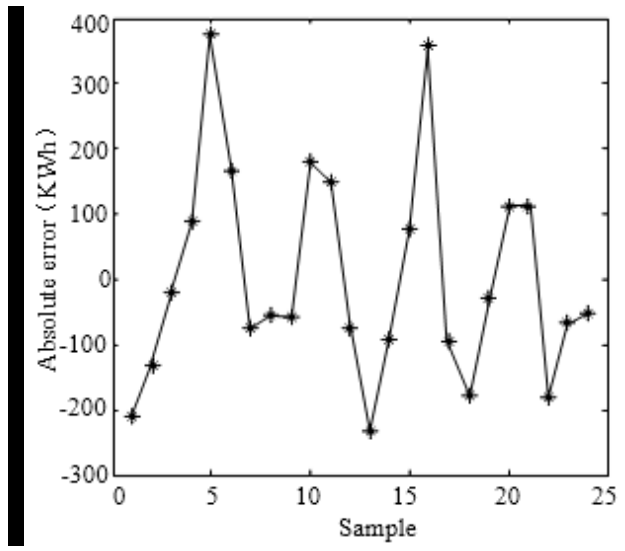

Figure 5. Finite GA- BP error diagram

Standard BP neural network and GA-BP neural network prediction errors are shown in Fig.4 and Fig.5. The absolute error of standard BP neural network to predict the power demand is large, and the maximum absolute error is $1544 \mathrm{KWh}$. The absolute error of GA-BP neural network to predict the power demand is small, and the maximum absolute error is $377 \mathrm{KWh}$. The results show that the prediction ability of GA-BP neural network is better than the standard BP neural network.

The relative errors of standard BP neural network and GA-BP neural network are shown in Fig. 6 and Fig. 7 Standard BP neural network prediction relative error range from $-10 \%$ to $15 \%$. GA-BP neural network prediction relative error range from $-4 \%$ to $4 \%$. The results show that GA-BP neural network has good stability and high precision in power demand forecasting. 


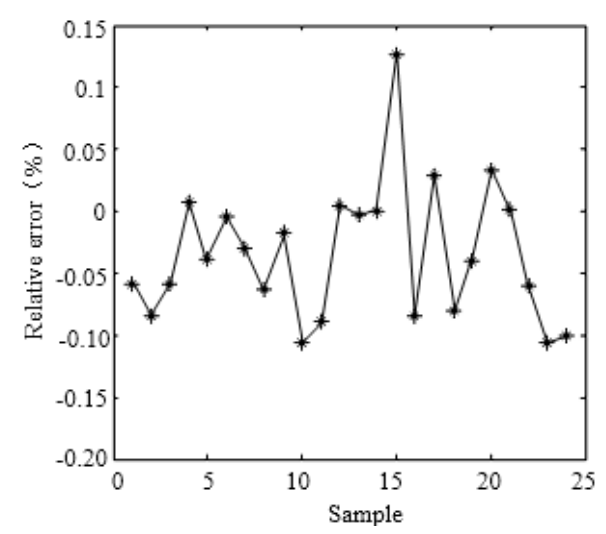

Figure 6. Finite Standard BP relative error diagram

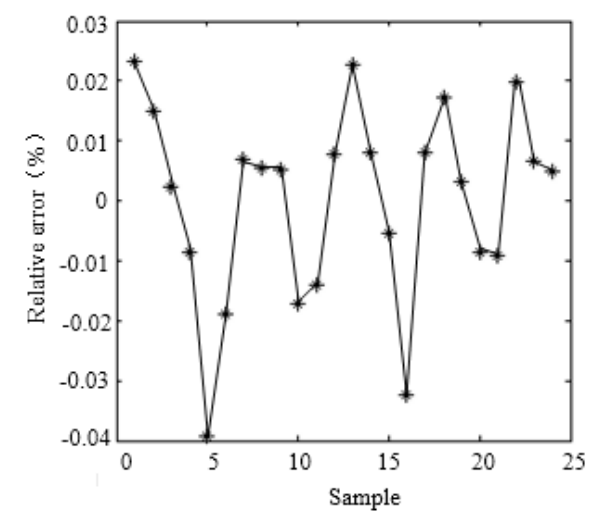

Figure 7. Finite GA- BP relative error diagram

\section{Conclusions}

EMS system has the functions of monitoring and management, energy consumption data analysis and prediction, energy network optimization. GA-BP neural network prediction model relative error range $-4 \%$ to $4 \%$, it has high prediction accuracy. EMS can improve the management of energy consumption and achieve the purpose of energy saving. This paper has a high reference and practical significance.

\section{References}

[1] DING Ming-qi, CAO Hai-bing. Practice and Exploration of Energy Management System in Pulp and Paper Enterprises [J]. China Pulp \& Paper Industry, 2016, 37(9) : 79-82.

[2] NING Kui-wei, LI Ming-hui. Control Parameter Tuning Based on Genetic Algorithm and Its Application in Temperature Control [J]. Packaging Engineering, 2016, 37(5) : 141-144.

[3] YE Nuo, LI Ji-geng, HONG Meng-na. Analysis of Energy Consumption and Energy Saving Potential Based on the Monthly Data of Enterprises in Paper Industry [J]. China Pulp \& Paper , 2016, 35(8) : 35-39.

[4] LI Wei-jiang, MA Yong-wen. Predicting the Papermaking Wastewater Treatment Process Based on Genetic Algorithm and Neural Network [J]. Transactions of China Pulp \& Paper , 2010, 25(1) : 67-70.

[5] WANG Xiao-yao, SU Wei-jun, YU Chong-chong. The Implementation of Paper Mill Energy Management System [J].Paper Science \& Technology, 2014, 33(6) : 140-145.

[6] B.data Energy Management[DB/OL].

http://w5.siemens.com/italy/web/IndustryGreenProgram/greenindustria/risparmioenergia/automazin dustriale/Documents/b.data_brochure_A4_eng_OK_low.pdf, 2013. 
[7] LI Hong-wei, YANG Guo-wei. Predicting Gas-oil Ratio of Original Oil by Fractional Linear Neural Network Based on Genetic Algorithm [J].Value Engineering, 2013, 28(2): 221-226.

[8] LI Zhen-bo, LI Ji-geng. Study on Energy Saving Optimization of Vacuum System of Paper Machine [J]. China Pulp \& Paper, 2016, 35(9): 8-11. 